Ann. Génét. Sél. anim., 1977, 9 (1), 105-112.

\title{
Variation de la différentielle phénotypique de sélection $\left({ }^{1}\right)$
}

\author{
J. RAZUNGLES \\ Station d'Amélioration génétique des Animaux, \\ Centre de Recherches de Toulouse, I.N.R.A., \\ 3 I 320 Castanet-Tolosan
}

\section{Résumé}

Dans certains plans de sélection pour lesquels le nombre d'individus soumis à sélection ou le nombre d'individus sélectionnés est faible, l'étude de l'écart-type dè la différentielle phénotypique de sélection revêt une importance particulière. Nous avons étudié la variation de cette différentielle et montré comment son écart-type passait par un maximum lorsque le nombre d'individus soumis à sélection était compris entre 5 et io fois le nombre d'individus sélectionnés.

\section{Introduction}

De nombreuses études ont visé à maximiser le gain génétique attendu par unité de temps, dans le cadre de recherches d'optimisation génétique de plans d'amélioration. Si la valeur génétique des reproducteurs est estimée par un index (régression de la valeur génétique sur les valeurs des variables phénotypiques prédictrices), ces études considèrent l'espérance du progrès génétique suite à la différentielle de sélection pratiquée sur l'index.

Nous avons montré (RAzUngLes, I974) l'intérêt de considérer aussi la fluctuation du progrès génétique réalisé. HiLl (I969) avait tenu compte de cette fluctuation dans l'analyse des résultats d'expérience de sélection en vue d'estimer les paramètres génétiques. Nous avions alors cité deux sources principales de fluctuation du progrès génétique :

- L'aléa sur la réponse génétique que nous avons étudié.

- L'aléa sur la différentielle de sélection phénotypique que nous n'avions pas analysé.

(1) Cet article a fait l'objet d'une communication lors du séminaire sur " L'estimation de la valeur génétique des reproducteurs "organisé à Toulouse les 6 et 7 octobre 1976 par le Département de Génétique Animale de l'I.N.R.A. (France). 
L'objet de cette note est donc d'étudier l'incidence de la variance d'échantillonnage de l'intensité de sélection (BurRows, I975).

\section{I. - Position du problème}

Soit P la variable aléatoire représentant la valeur phénotypique d'un caractère. Cette variable a une moyenne $\mu$ et une variance $\sigma_{\mathbf{p}}^{2}$. Considérons les $s$ valeurs prises indépendamment par cette variable dans le cas d'un $s$-échantillon d'individus que l'on soumet à la sélection :

$$
\mathrm{P}_{1}, \mathrm{P}_{2}, \ldots, \mathrm{P}_{i}, \ldots, \mathrm{P}_{s}
$$

La sélection consiste à prendre un ensemble $\mathrm{R}$ de $n_{\delta}$ indices dans l'ensemble $[\mathrm{I}, 2, \quad s]$. Le choix de ces indices suppose que l'on ordonne les valeurs prises par $\mathrm{P}$. On range donc les $\mathrm{P}_{i}$ par ordre croissant pour obtenir :

$$
\mathrm{P}_{(1)} \leqslant \mathrm{P}_{(2)} \leqslant \ldots \leqslant \mathrm{P}_{(i)} \leqslant \ldots \leqslant \mathrm{P}_{(s)}
$$

Les variables ci-dessus ne sont plus indépendantes.

Soient les moyennes :

$$
\overline{\mathrm{P}}_{n_{s}}=\frac{\sum_{i \in \mathrm{R}} \mathrm{P}_{(i)}}{n_{s}} \text { et } \overline{\mathrm{P}}=\frac{\sum_{i=\mathrm{I}}^{s} \mathrm{P}_{(i)}}{s}=\frac{\sum_{i=1}^{s} \mathrm{P}_{i}}{s}
$$

La différentielle phénotypique de sélection est égale à

$$
\Delta \mathrm{P}=\overline{\mathrm{P}}_{n_{s}}-\overline{\mathrm{P}}
$$

On considère la valeur $\Delta \mathrm{P}=\overline{\mathrm{P}}_{n_{s}}-\mu=\Delta \mathrm{P}+(\overline{\mathrm{P}}-\mu)$

$$
\mathrm{E}(\mathrm{DP})=\mathrm{E}(\Delta \mathrm{P}) \text { car } \mathrm{E}(\overline{\mathrm{P}})=\mu
$$

On définit maintenant $k$ et $v$ :

$$
\mathrm{E}(\mathrm{DP})=k \sigma \quad \text { et } \quad \operatorname{var}(\mathrm{DP})=\frac{v \sigma_{\mathrm{P}}^{3}}{n_{s}}
$$

Dans le cas d'une variable $\mathrm{P}$ centrée et réduite, soit si $\mu=0$ et $\sigma_{3}^{2}=\mathrm{I}$

$$
n_{s}=\sum_{i \varepsilon \mathbf{R}} \mu_{i, s} \quad n_{s} v=\sum_{i \varepsilon \mathrm{R}} \sum_{j \in \mathrm{R}} \sigma_{i j, s}
$$

où

$$
\mu_{i, s}=\mathrm{E}\left(\mathrm{P}_{(i)}\right) \text { et } \sigma_{i j, s}=\operatorname{Cov}\left(\mathrm{P}_{(i)}, \mathrm{P}_{(j)}\right)=\mathrm{E}\left(\mathrm{P}_{(i)} . \mathrm{P}_{(j)}\right)
$$

Dans ce cas : $\mathrm{E}(\Delta \mathrm{P})=\mathrm{E}(\mathrm{DP})=k$ et $\operatorname{var}(\mathrm{DP})=\frac{v}{n_{s}}$.

Nous considérons dorénavant le cas de variables normales.

Les valeurs considérées ici, $P_{(i)}-\overline{\mathrm{P}}$, sont des écarts à la moyenne. 
Ce sont des valeurs provenant d'un échantillon de variables aléatoires de moyennes nulles, des variances égales à :

$$
\frac{(s-\mathrm{I}) \sigma_{\mathrm{P}}^{2}}{s} \text { et de corrélations deux à deux égales à }-\frac{s-\mathbf{I}}{\mathrm{I}} \text {. }
$$

A partir d'équations déterminées par OwEN et STECK (I962) on montre que :

$$
n_{s} \operatorname{var}\left(\frac{\Delta \mathrm{P}}{\sigma_{\mathrm{P}}}\right)=v-\frac{n_{s}}{s} \text { et } \operatorname{var}(\Delta \mathrm{P})=\operatorname{var}(\mathrm{DP})-\frac{\sigma_{\mathrm{P}}^{2}}{s} .
$$

Considérons le cas où la sélection retient les $n_{8}$ valeurs les plus élevées ou les $n_{s}$ valeurs les plus basses. Il suffit de considérer les $n_{s}$ plus élevées définies par

$$
\mathbf{R}=\left\{\left(s-n_{s}+\mathrm{I}\right),\left(s-n_{s}+2\right), \ldots \ldots,(s)\right\}
$$

puisque pour les $n_{s}$ plus faibles, seul le signe de $k$ change et $v$ est inchangée.

Soit $\alpha=\frac{n_{8}}{s}$ la proportion d'individus sélectionnés.

On remplacera $k$ par $k_{\alpha}$ et $v$ par $v_{\alpha}$. Soit $f$ la densité et $\mathrm{F}$ la fonction de répartition d'une loi normale centrée réduite.

\section{II. - Propriétés de $\mathbf{v}_{\alpha}$}

Burrows (1975) a montré que $\alpha\left(\mathrm{I}-v_{\alpha}\right)=(\mathrm{I}-\alpha)\left(\mathrm{I}-v_{1-\alpha}\right)$.

Cette relation n'est valable que dans le cas de lois normales. Il suffit alors de ne calculer $v_{\alpha}$ que pour $o<\alpha<0,5$, et l'on a l'éventail de toutes les valeurs de la variance de la différentielle de sélection.

Quand la taille $s$ de l'échantillon augmente, après une légère décroissance initiale, $v_{\alpha}$ tend en croissant vers une valeur asymptotique (FINNEY, 1956)

$$
\widehat{v}_{\alpha}=\widehat{\sigma}_{\alpha}^{2}+(\mathrm{I}-\alpha)\left(\widehat{k}_{\alpha}-x_{\alpha}\right)^{2}
$$

où $\widehat{\sigma_{\alpha}^{2}}$ est la variance d'une $\mathrm{N}(\mathrm{O}, \mathrm{I})$ après troncature pour les valeurs supérieures à $x_{\alpha}$, soit

$$
\widehat{\sigma}_{\alpha}^{2}=\mathrm{I}-\widehat{k}_{\alpha}\left(k_{\alpha}-x_{\alpha}\right) \text { FINNEY (I956) }
$$

On peut montrer, en utilisant la fonction $\mathrm{L}(u)=f(u)+u \mathrm{~F}(u)$ définie initialement par GRUNDY el al. (I956), et en mettant $\widehat{v}_{\alpha}$ sous la forme $\widehat{v}_{\alpha}=\mathrm{I}-\left(\widehat{k}_{\alpha}-x_{\alpha}\right)$ $\mathrm{L}\left(x_{\alpha}\right)$ que $\alpha \leqslant v \leqslant \bar{v}_{\alpha} \leqslant \alpha+(\mathrm{I}-\alpha)\left(\mathrm{I}-\frac{2}{\pi}\right)$.

La borne inférieure peut-être affinée empiriquement.

$$
\text { III. - Variations de } \operatorname{Var} \frac{\Delta \mathbf{P}}{\sigma \boldsymbol{p}}=\frac{\mathrm{V} \alpha-\alpha}{n_{s}}
$$

La variance de la différentielle de sélection par unité d'écart-type phénotypique se présente sous la forme d'un quotient. 
TABLEAU I

\begin{tabular}{|c|c|c|c|c|c|c|c|c|}
\hline \multicolumn{2}{|c|}{$\begin{array}{c}\alpha \% \\
\text { de sélectionnés }\end{array}$} & \multicolumn{7}{|c|}{ Nombre d'individus sélectionnés } \\
\hline$\alpha$ & $v_{\alpha}-\alpha$ & I & 2 & 3 & 4 & 5 & Io & 20 \\
\hline I & 0,178 & 0,422 & 0,298 & 0,244 & 0,2 I I & 0,189 & $0, \mathbf{1} 33$ & 0,094 \\
\hline 2 & 0,204 & $0,45^{2}$ & 0,319 & $0,26 I$ & 0,226 & 0,202 & 0,143 & O,IOI \\
\hline 3 & 0,224 & 0,473 & 0,335 & 0,273 & 0,237 & 0,212 & $0,5^{\circ}$ & 0,106 \\
\hline 4 & 0,235 & $0,4^{8} 5$ & 0,343 & 0,280 & 0,242 & $0,2 \mathrm{I} 7$ & o, I 53 & 0,108 \\
\hline 5 & 0,246 & 0,496 & $0,35 \mathrm{I}$ & 0,286 & $0,24^{8}$ & 0,222 & o, I 57 & O, I I I \\
\hline 6 & 0,255 & 0,505 & 0,357 & 0,292 & 0,252 & 0,226 & 0,160 & O, I I 3 \\
\hline 7 & 0,260 & 0,510 & $0,36 \mathrm{I}$ & 0,294 & 0,255 & 0,228 & $0, \mathrm{I} 6 \mathrm{I}$ & $0, x_{4} 4$ \\
\hline 8 & 0,263 & $0,5^{1} 3$ & 0,363 & 0,296 & 0,256 & 0,229 & 0,162 & o, I I 5 \\
\hline 9 & 0,265 & 0,515 & 0,364 & 0,297 & 0,257 & $0,23^{\circ}$ & 0,163 & 0,115 \\
\hline ro & 0,266 & 0,516 & 0,365 & 0,298 & $0,25^{8}$ & $0,23 \mathrm{I}$ & 0,163 & 0,115 \\
\hline II & 0,268 & $0,5 \times 8$ & 0,366 & 0,299 & 0,259 & 0,232 & 0,164 & 0,116 \\
\hline 12 & 0,269 & 0,519 & 0,367 & 0,299 & 0,259 & 0,232 & 0,164 & 0,116 \\
\hline I3 & 0,269 & 0,519 & 0,367 & 0,299 & 0,259 & 0,232 & 0,164 & 0,116 \\
\hline 14 & 0,268 & 0,518 & 0,366 & 0,299 & 0,259 & 0,232 & 0,164 & 0,116 \\
\hline I 5 & 0,268 & 0,518 & 0,366 & 0,299 & 0,259 & $0,23^{2}$ & 0,164 & $0, \mathrm{II} 6$ \\
\hline 16 & 0,269 & 0,519 & 0,367 & 0,299 & 0,259 & 0,232 & 0,164 & 0,116 \\
\hline I 7 & 0,269 & 0,519 & 0,367 & 0,299 & 0,259 & 0,232 & 0,164 & 0,116 \\
\hline I 8 & 0,268 & 0,518 & 0,366 & 0,299 & 0,259 & 0,232 & 0,164 & 0,116 \\
\hline 19 & 0,267 & 0,517 & 0,365 & 0,298 & 0,258 & $0,23 \mathrm{I}$ & 0,163 & 0,116 \\
\hline 20 & 0,266 & 0,516 & 0,365 & 0,298 & $0,25^{8}$ & 0,231 & 0,163 & $U, 116$ \\
\hline 21 & 0,264 & 0,514 & 0,363 & 0,297 & 0,257 & 0,230 & 0,162 & 0,115 \\
\hline 22 & 0,262 & 0,512 & 0,362 & 0,296 & $0,25^{6}$ & 0,229 & $\mathrm{c}, \mathrm{I} 62$ & $0,1 \times 4$ \\
\hline 23 & 0,260 & 0,510 & $0,36 \mathrm{I}$ & 0,294 & 0,255 & 0,228 & $0, \mathrm{I} 6 \mathrm{I}$ & 0,114 \\
\hline 24 & 0,257 & 0,507 & $0,35^{8}$ & 0,293 & 0,253 & 0,227 & 0,160 & $0,1 \mp 3$ \\
\hline 25 & 0,255 & 0,505 & 0,357 & 0,292 & 0,252 & 0,226 & 0,160 & $0, \mathrm{I} \mathbf{I} 3$ \\
\hline 26 & 0,253 & 0,503 & $0,35^{6}$ & 0,290 & $0,25 \mathrm{I}$ & 0,225 & o, I 59 & 0,112 \\
\hline 27 & 0,250 & 0,500 & 0,354 & 0,289 & $0,25^{\circ}$ & 0,224 & o, I $5^{8}$ & O, I I 2 \\
\hline 28 & 0,247 & 0,497 & $0,35^{\mathrm{I}}$ & 0,287 & 0,248 & 0,222 & o, I 57 & $0, I \mid I I$ \\
\hline 29 & 0,245 & 0,495 & $0,35^{\circ}$ & 0,286 & 0,247 & $0,22 \mathrm{I}$ & o, I 57 & O, I I I \\
\hline 30 & 0,242 & 0,492 & $0,34^{8}$ & 0,284 & 0,246 & 0,220 & 0,156 & $0, \mathrm{I} I 0$ \\
\hline 35 & 0,229 & 0,479 & $0,33^{8}$ & 0,276 & 0,239 & $0,2 \mathrm{I}_{4}$ & $0, I_{5}$ I & 0,107 \\
\hline 40 & 0,2 I 3 & 0,462 & 0,326 & 0,266 & 0,231 & 0,206 & 0,146 & 0,103 \\
\hline 45 & 0,197 & 0,444 & $0,3 I_{4}$ & 0,256 & 0,222 & 0,198 & $0, \mathrm{I}_{4} \mathrm{O}$ & 0,099 \\
\hline 50 & 0,179 & 0,423 & 0,299 & 0,244 & 0,212 & 0,189 & 0,134 & 0,095 \\
\hline 55 & 0,161 & $0,40 I$ & 0,284 & 0,232 & $0,20 \mathrm{I}$ & 0,179 & 0,127 & 0,090 \\
\hline 60 & $0, I_{42}$ & 0,377 & 0,266 & $0,2 \mathrm{I} 8$ & 0,188 & 0,169 & 0,119 & 0,084 \\
\hline 65 & 0,123 & $0,35 \mathrm{I}$ & $0,24^{8}$ & 0,202 & 0,175 & 0,157 & O, I I I & 0,078 \\
\hline 70 & $0, \mathrm{IO}_{4}$ & 0,322 & 0,228 & 0,186 & 0,161 & 0,144 & 0,102 & 0,072 \\
\hline 75 & $0,08_{4}$ & 0,290 & 0,205 & 0,167 & 0,145 & 0,130 & 0,092 & 0,065 \\
\hline 80 & 0,065 & 0,255 & $0, \mathrm{I} 80$ & 0,147 & 0,127 & o, I I 4 & $0,08 \mathrm{I}$ & 0,057 \\
\hline 85 & 0,047 & 0,217 & 0,153 & 0,125 & 0,108 & 0,097 & 0,069 & 0,048 \\
\hline 90 & 0,029 & 0,170 & 0,120 & 0,098 & 0,085 & 0,076 & 0,054 & $0,03^{8}$ \\
\hline 95 & 0,013 & $0,1 \mathbf{r}_{4}$ & $0,08 \mathrm{I}$ & 0,066 & 0,057 & $0,05 \mathrm{I}$ & 0,036 & 0,025 \\
\hline IOO & 0,000 & 0,000 & 0,000 & 0,000 & 0,000 & 0,000 & 0,000 & 0,000 \\
\hline
\end{tabular}

SCHAEFFER et al. (I970) ont montré que le numérateur $v_{\alpha}-\alpha$ variait très peu en fonction de la taille de l'échantillon lorsque le pourcentage $\alpha$ de sélectionnés était fixé. 
Par conséquent on peut remarquer que pour $\sigma$ fixé, $\operatorname{Var} \frac{\Delta P}{\sigma_{P}}$ est pratiquement inversement proportionnelle au nombre d'individus sélectionnés. mation

Des calculs analytiques ne peuvent être effectués qu'en prenant l'approxi-

$$
v \alpha \simeq v \alpha \simeq I-(I-\alpha) \frac{2}{\pi}
$$

valable seulement lorsque $\alpha>0,3$.

Nous avons étudié la variation de $\sqrt{\operatorname{Var} \frac{\Delta \mathrm{P}}{\sigma_{\mathbf{P}}}}$ quand $\alpha$ varie pour $n_{s}$ fixé fixé et cela pour un certain nombre de valeurs de $n_{s}$ (tableau I, fig. I) en utilisant l'approximation de SchaEfFER et al. (r97o).

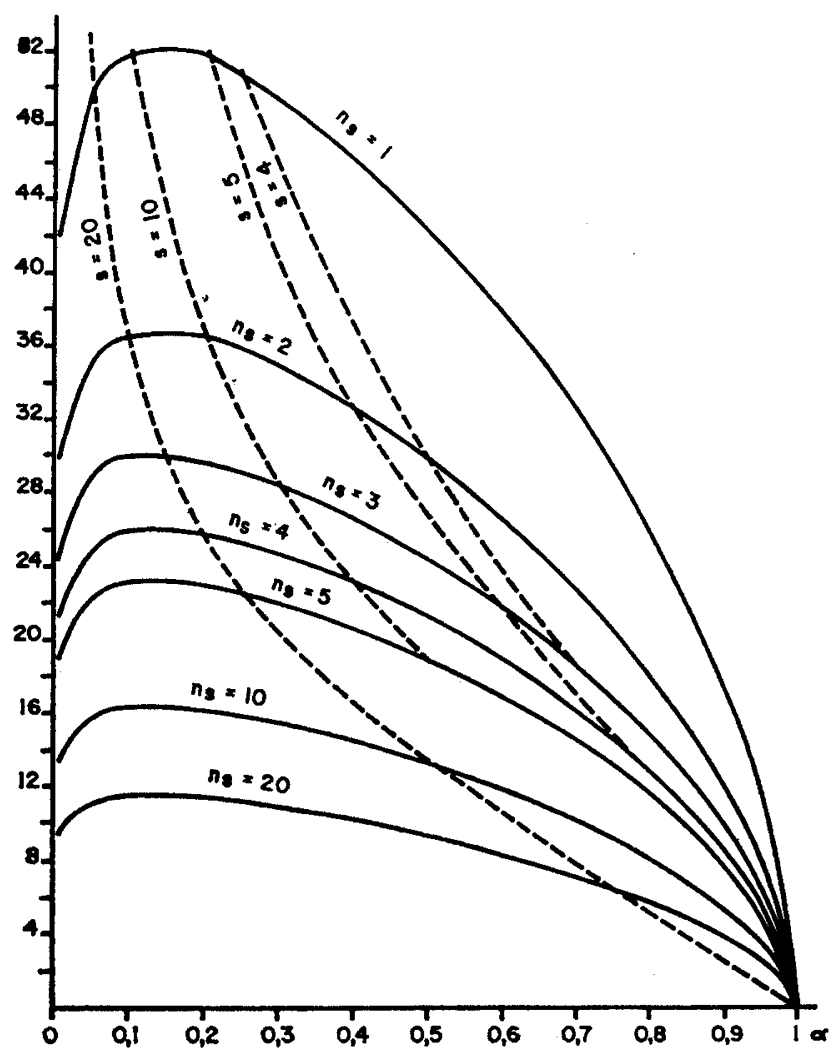

FIG. I. - Variations de $\sqrt{\frac{V \alpha-\alpha}{n_{s}}}=\sqrt{\operatorname{var}\left(\frac{\Delta P}{\sigma_{p}}\right)}$ en fonction de $\alpha$.

Variation of $\sqrt{\frac{V \alpha-\alpha}{n_{s}}}=\sqrt{\operatorname{var}\left(\frac{\Delta P}{\sigma_{p}}\right)}$ in terms of $\alpha$. 


\section{IV. - Application biologique (sélection sur descendance des lapins mâles)}

Nous avons considéré le même exemple que dans Razungles (1974) décrit dans Poujardieu et al. (I974).

Le nombre de mâles sélectionnés $\left(n_{\delta}\right)$ est égal à 5 . Le plan d'accouplement est tel que 25 mâles sont accouplés chacun à 3 femelles.

L'écart-type de la différentielle de sélection est égal à

$$
\sqrt{\operatorname{Var} \Delta \mathbf{P}}=\sqrt{\operatorname{Var} i} . \sigma_{p}=0,23 \sigma_{p}
$$

$i=$ intensité de sélection.

Pour les caractères envisagés, gain moyen quotidien et poids à 77 jours, on obtient :

\begin{tabular}{|c|c|c|c|}
\hline & $h^{2}$ & $\mathrm{E}(\Delta \mathrm{G})$ & $\sigma \Delta p$ \\
\hline $\begin{array}{l}\text { G.M.Q. • • } \\
\text { Poids à } 77 \text { jours }\end{array}$ & $\begin{array}{l}0,6 \mathrm{I} \\
0,55\end{array}$ & $\begin{array}{r}3,4 \\
180,7\end{array}$ & $\begin{array}{r}0,5 \\
32,6\end{array}$ \\
\hline
\end{tabular}

Ces premiers résultats montrent l'importance de la variation de la différentielle phénotypique de sélection d'autant plus que le cas envisagé se situe au maximum de la variation de cette différentielle.

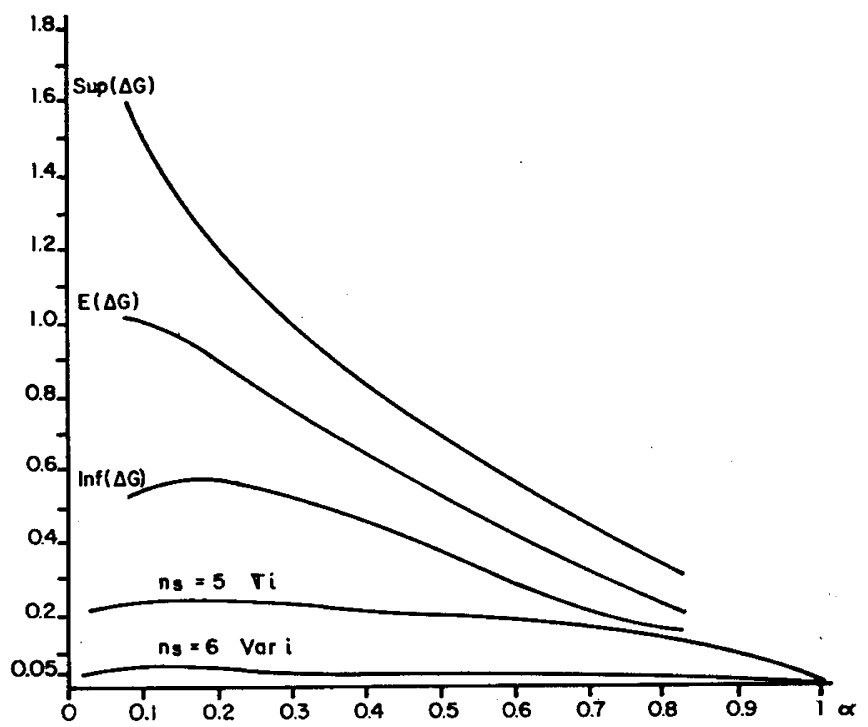

IIG. 2. - Intervalle de confiance du progrès génétique et écart-type de la différentielle phénotypique de sélection dans le cas oì $\mathrm{h}^{2}=0,6 ; \mathrm{n}_{\delta}=5 ; 6$ descendants par femelle; un nombre total fixé de femelles égal à $\mathbf{2} 0$; seuil de o, ro.

Confidence interval of genetic progress and standard deviation of phenotypic of selection differential for $\mathrm{h}^{2}=0,6 ; \mathrm{n}_{s}=5 ; 6$ progenies by female; a total fixed number of females of 120 ; level of $\mathrm{O}, \mathrm{IO}$. 
D'autre part nous avons traité le cas d'un nombre de mâles sélectionnés de $n_{s}=5$ et fait varier le nombre de mâles soumis à la sélection, c'est-à-dire le pourcentage d'individus sélectionnés (fig. 2). La courbe obtenue a été inscrite sur le même graphique que celui de l'intervalle de confiance précédemment calculé du progrès génétique dans le cas d'une population constante de femelles $(s d=\mathrm{I} 2 \mathrm{O})$ et pour un caractère d'héritabilité égal à 0,6 .

On ne peut à nouveau que constater l'importance de la variation de l'écart type de l'intensité de sélection qui se situe entre I5 $_{5}$ et 20 p. Ioo de l'écart type phénotypique.

Ces calculs numériques montrent que $\frac{v_{\alpha}-\alpha}{n_{s}}$ passe par un maximum lorsque le pourcentage de sélectionnés est compris entre Io et $20 \mathrm{p}$. Ioo soit lorsque la taille $s$ de l'échantillon est telle que $5 n_{s}<s<$ Io $n_{s}$.

La variation, très forte quand le nombre de sélectionnés est très bas, s'estompe peu à peu pour devenir relativement faible quand la taille de l'échantillon est supérieure à 20 .

Il faut remarquer que ces courbes ne sont pas à prendre en considération sur toute leur longueur car la taille de l'échantillon $s=\frac{\alpha}{n_{s}}$ doit être un nombre entier.

- $\alpha$ étant constant, lorsque $n_{s}$ varie, $s$ varie également. Si l'on veut stigmatiser ce dernier type de variation, on remarquera que, si l'on fixe $s$ et si l'on fait varier $\alpha$, on observe une décroissance très rapide de la variance de la différentielle de sélection.

\section{Conclusion}

Burrows (I975) avait considéré uniquement la variance de la moyenne des phénotypes des individus sélectionnés et n'avait pas considéré les applications possibles en amélioration génétique.

Ces premiers résultats indiquent qu'il semble être particulièrement important d'étudier l'écart-type de la différentielle de sélection, de façon à mieux connaître la précision du progrès génétique attendu. De plus, sa prise en considération pourrait modifier, semble-t-il, les résultats des deux sources de variation du progrès génétique que nous avons considérées précédemment (RAZUNGLEs I974). Il semble donc utile d'envisager maintenant de façon globale, compte tenu de la variation du progrès, l'optimisation génétique des plans d'amélioration, et trouver un compromis pour résoudre l'antagonisme suivant :

- le nombre de sélectionnés doit être petit pour maximiser l'espérance du progrès génétique mais, par contre, s'il est petit, la différentielle de sélection a une grande variance.

Reçu pour publication en février 1977.

\section{Summary}

\section{Variation of phenotypic selection differential}

In some selection schemes for which the number of measured individuals or the number of selected individuals is low, the study of the standard deviation of the phenotypic selection differential is very important. We have studied the variation of this differential and shown how its standard deviation has a maximum when the number of measured individuals is between 5 and to times the number of selectionned individuals. 


\section{Références bibliographiques}

Burrows P. M., 1972. Expected selection differentials for directional selection. Biometrics, 28, IO9I-IIOO.

BurRows P. M., I975. Variance of selection differentials in normal samples. Biometrics, 31, I 25-I33.

FINNEY D. J., 1956. The consequences of a selection for a variate subject to errors of measurement. Inst. Int. de Stat., 24, I-10.

Grundy P. M. G., Healy M. J. R. et Rees D. H. R., 1956. Economics choice of the amount of experimentation. J. Roy. Statis. Soc. B, 18, 32-49.

HrLL W. G., I969. On the theory of artificial selection in finite population. Genet. Res. Camb., $13, \mathrm{I}_{43}-63$.

Owen D. B. et Steck G. P., 1962. Moments of order statistics from the equicorrelated multivariate normal distribution. Ann. Math. Statis., 33, r286-9r.

Poujardieu B., Rouvier R., Vrillon J. L. et Donal R., I974. Modèle de la sélection du lapin sur les caractèves de croissance et d'efficacité alimentaire. $\mathbf{I}^{\mathrm{er}}$ Congrès Mondial de Génétique appliquée à la production animale, Madrid, 3, 497-504.

Razungles J., I974. Espérance et précision du progrès génétique. Ann. Génét. Sél. anim., 6. 4I 5-426.

Sales J. et Hili W. G., I 976 . Effect of sampling errors on efficiency of selection indices. I. Use of information from relatives for single trait improvement. Anim. Product., 22, I-I7. II. Use of information on associated traits for improvement of a single important trait. Anim. Product. 23, I-I4.

Schaeffer L. R., VAN Vleck L. D. et Velasco J. A., I97o. The use of order statistics with selected records. Biometrics, 26, 854-859.

Williams J. S., 1962. The evaluation of a selection index. Biometrics, 18, 375-93. 